\title{
RESEARCH PAPER \\ DEFLUORINATION OF DRINKING WATER USING SURFACTANT MODIFIED ZEOLITES
}

\author{
E. A. Donkor ${ }^{1}$, R. Buamah ${ }^{2}$ and B. Kwakye-Awuah ${ }^{3}$ \\ ${ }^{I}$ Department of Food Science and Technology, KNUST, Kumasi \\ E-mail: estheradonkor@gmail.com \\ ${ }^{2}$ Department of Civil Engineering, KNUST, Kumasi \\ E-mail: rbuamah@gmail.com \\ ${ }^{3}$ Department of Physics, KNUST, Kumasi \\ E-mail:brikye2000@yahoo.com
}

\begin{abstract}
The prevalence of high concentrations of fluoride (i.e. $>1.5 \mathrm{mg} / \mathrm{l}$ ) in groundwater in the Northern part of Ghana especially around the Bongo communities has been an issue of concern. Owing to the arid nature of these localities, the inhabitants (who are mainly peasant farmers), rely more on groundwater sources for their drinking water. With the strenuous nature of their job and the warm weather conditions, the farmers generally consume more water, thus becoming more vulnerable to dental and skeletal fluorosis. This study focused on the removal of fluoride from groundwater by employing surfactant modified zeolites (SMZ) synthesized using locally available kaolin material as precursor. The zeolite synthesis involved calcination of kaolin, alkaline fusion and hydrothermal treatment. The final product was modified with $5 \mathrm{~g} / \mathrm{L} \mathrm{Hexadecyl-}$ trimethylammonium bromide (HDTMABr). The zeolite was characterised by Xray Diffraction (XRD), Energy Dispersive Xray (EDX) and Scanning Electron Microscopy (SEM) and the modified form employed in batch fluoride removal studies. The fluoride adsorption kinetics was studied using model water with varying initial fluoride concentration. From the EDX analysis, the synthesized zeolite NaLSX was found to comprise predominantly Oxygen (60\%), Silicon (15\%), and Aluminium (13\%). The SEM showed the zeolite NaLSX crystals to be octahedrally shaped. The unmodified zeolite NaLSX was incapable of adsorbing fluoride ions but the surfactant modified zeolite adsorbed fluoride. The fluoride adsorption capacity of the modified zeolite was pH dependent and peaked at pH 6.0 - 7.0.
\end{abstract}

Keywords: characterization, defluoridation, groundwater, surfactant, zeolites

\section{INTRODUCTION}

Groundwater is often presumed to be fairly safe for consumption compared to surface water because the soil acts as a big filtering system straining off most of the suspended solids that otherwise would have contaminated it. This claim, however, is not always the case owing to the fact that groundwater at times has contaminants such as fluoride, arsenic, iron, calcium, magnesium, chromium etc. originating from geological formations that may impact negatively on the health of consumers (Cobbina et 
al., 2012).

Globally, fluoride is known to be a natural contaminant of groundwater resources (Murugan and Subramanian, 2006). The most affected regions of fluoride contamination in Ghana are the Upper West, Upper East and Northern Regions. Fluoride is essential for the building of healthy teeth and inhibiting dental carries among others. However, consumption of water with fluoride concentration above $1.5 \mathrm{mg} / \mathrm{l}$, the W.H.O. guideline value, can lead to acute and or chronic dental fluorosis. Exposure to very high fluoride (> $10 \mathrm{mg} \mathrm{F}^{-} /$day) over a prolonged period (i.e. 10 to 20 years) can result in acute to chronic skeletal fluorosis. Taking cognizance of the fact that both dental and skeletal fluorosis are irreversible conditions, it is expedient that in situations where fluoride concentration exceeds the acceptable limit of $1.5 \mathrm{mg} / 1$, defluoridation methods are devised to reduce or totally eradicate the risk of fluoride poisoning (Renuka and Pushpanjali,2013). It is against this background that, this study focused on the synthesis of surfactant modified zeolites formed from kaolin and its ability to defluoridate water.

\section{MATERIALS AND METHODOLOGY}

The kaolin was collected from Wassa in the Western Region of Ghana and it had 54.8\% kaolinite and $46.2 \%$ quartz based on quantitative X-ray Diffraction analysis. All chemicals used in the study were of analytical grade.

The method used for the zeolite synthesis was obtained from studies reported by Shoumkova (2011); Reyes and Fiallo (2011), Keka et al. (2004) with some modifications. A preweighed quantity of kaolin was calcined and then alkaline fused with $\mathrm{NaOH}$ in the furnace at $600^{\circ} \mathrm{C}$ for an hour. It was then ground into fine powder after it had cooled. Water was added in a predetermined ratio and mixed thoroughly for some minutes. The resultant slurry from the mixture was transferred into Teflon bottles and heated at $80^{\circ} \mathrm{C}$ in an oven for 24 hours. It was then quenched in cold water. The product was filtered, rinsed with distilled water and dried in the oven at $105^{\circ} \mathrm{C}$ overnight. The surfactant modified zeolite was produced by treating synthesized zeolite with 5g/l HDTMA-Br solution in the proportions of 1:100 (solid to liquid) in a flocculator. The final product was rinsed and dried afterwards.

In order to ascertain the crystal structure and the composition of the synthesized zeolite, it was essential to characterize the zeolite. The Xray powder diffraction patterns of the zeolite samples were recorded on a Philips PW1710. Data processing was carried out using Philips APD software with a search/match facility and an ICDD database on a DEC Microvax minicomputer interfaced to the diffractometer. The morphology of the zeolite sample was then examined using the scanning electron microscope (ZEISS EVO50) equipped with energy dispersive X-ray spectrometer (EDX) (Zeiss, UK).

Batch adsorption experiments were performed using both unmodified and surfactant (HDTMA -Br) modified zeolites in a flocculator. Fluoride solutions $(5,10,15 \mathrm{mg} / \mathrm{L})$ were used in each experiment. A blank was prepared and run under similar conditions but without the adsorbents. The various experimental reactions were initiated by adding the adsorbents to the fluoride solutions and stirred continuously at 80 revolutions per minute (rpm). The solution in each beaker was sampled at pre-determined time intervals and immediately filtered. Each filtered sample was analysed for fluoride with an accumet ${ }^{\circledR}$ model 25 ion meter and a Mettler Toledo AG $\mathrm{pH}$ meter model was used for the $\mathrm{pH}$ measurements.

\section{RESULTS AND DISCUSSION Zeolite characterization}

The XRD pattern of the zeolites synthesized from the kaolin (Fig. 1) indicates that the main crystalline phase is zeolite Na-LSX. This indicates that a complete transformation of the kaolin to zeolite had taken place. From the EDX (fig. 2), the zeolite Na-LSX was found to comprise predominantly Oxygen (60\%), Silicon 


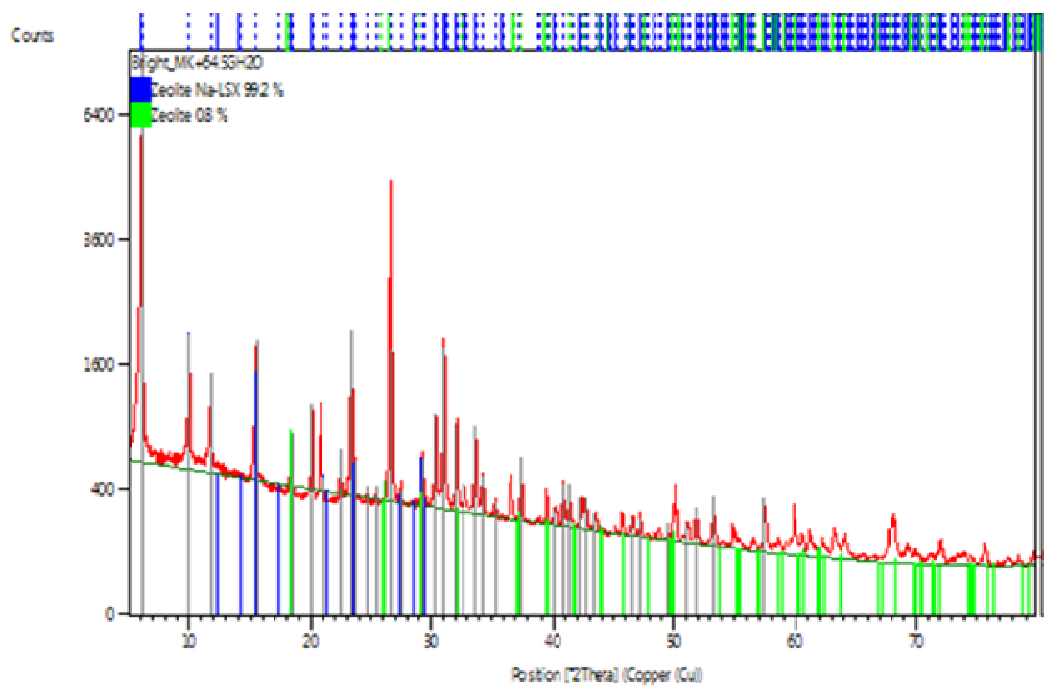

Fig. 1: XRD pattern of Zeolite Na-LSX

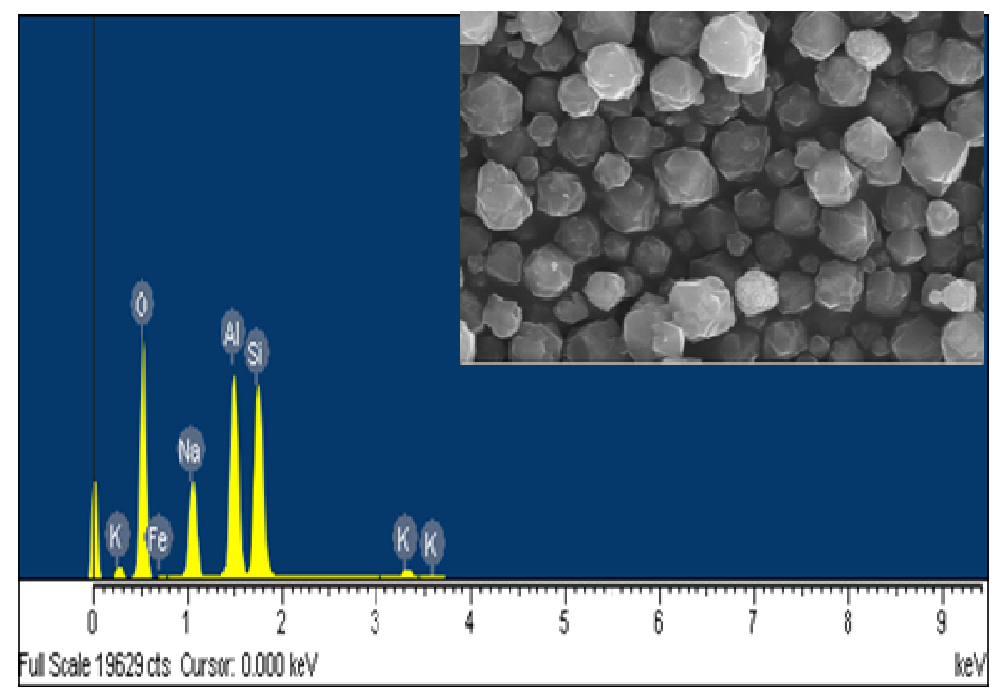

Fig 2: EDX and SEM spectrum of zeolite Na-LSX

(15\%) and Aluminium (13\%). Meanwhile sodium, potassium and iron were present as interlayer cations. From the SEM images the morphology of the zeolite Na-LSX was found to be octahedral.
Batch adsorption studies

From Fig. 3, it is observed that the unmodified zeolite did not show any capacity to remove fluoride. Generally, the surface charge of zeolites is negative (Mohammadi et al., 2012). 


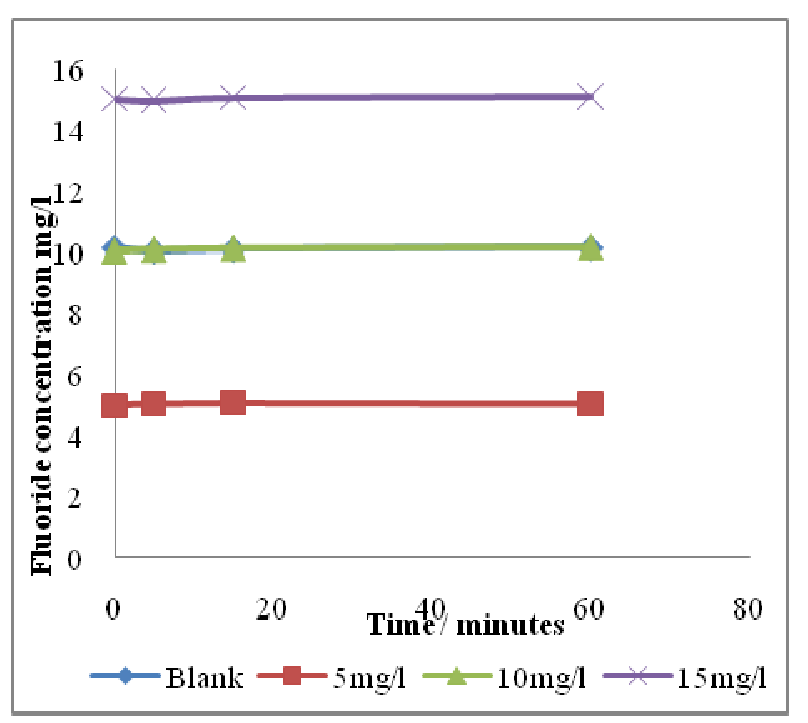

Fig. 3: Fluoride removal with unmodified zeolite Na-LSX

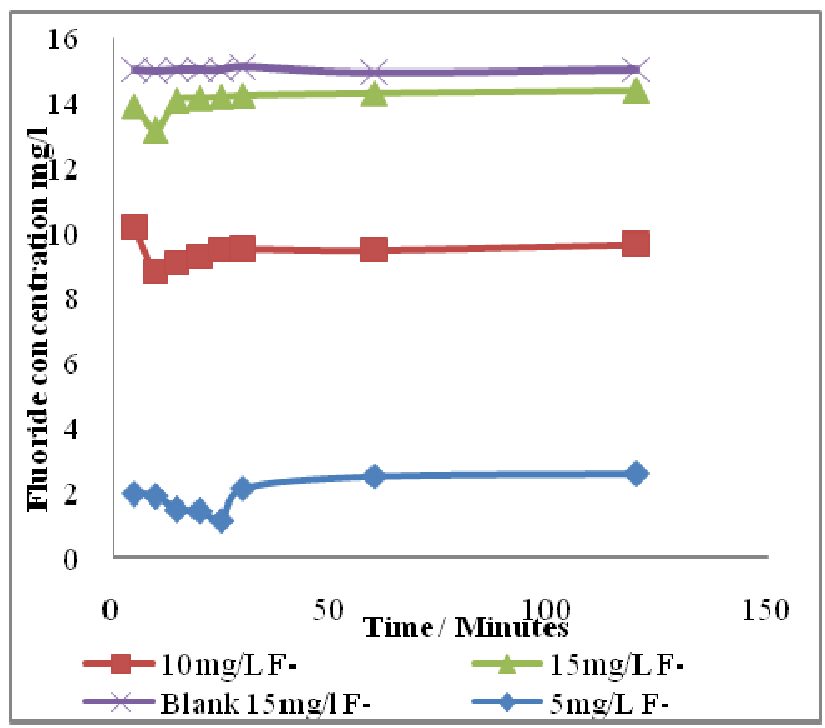

Fig. 4: Fluoride removal with HDTMA- Br modified zeolite under uncontrolled pH conditions

Fluoride being an electronegative element is attracted to positively charged ions therefore there was electrostatic repulsion by the negative charge on the surface of the zeolite. Hence the unmodified zeolites could not remove the fluoride from the water. For each of the fluoride concentrations in Fig. 4, the adsorbed fluoride leached into the solution after the initial drop 
observed around the $10^{\text {th }}$ and $25^{\text {th }}$ minutes. Monitoring the $\mathrm{pH}$ of the fluoride solution in the reaction vessel showed that the initial $\mathrm{pH}$ increased from 6.7 to 8.4 as the adsorption experiment proceeded. This increasing $\mathrm{pH}$ may have led to the leaching of fluoride adsorbed during the adsorption experiment (Fig. 4). A similar trend was reported in the work of Kaur et al. (2013). It can be observed from Fig. 5 that for the fluoride concentrations of $5 \mathrm{mg} / \mathrm{L}$ and $10 \mathrm{mg} / \mathrm{l}$, the fluoride removal was rapid in the first five minutes. Then fluoride removal

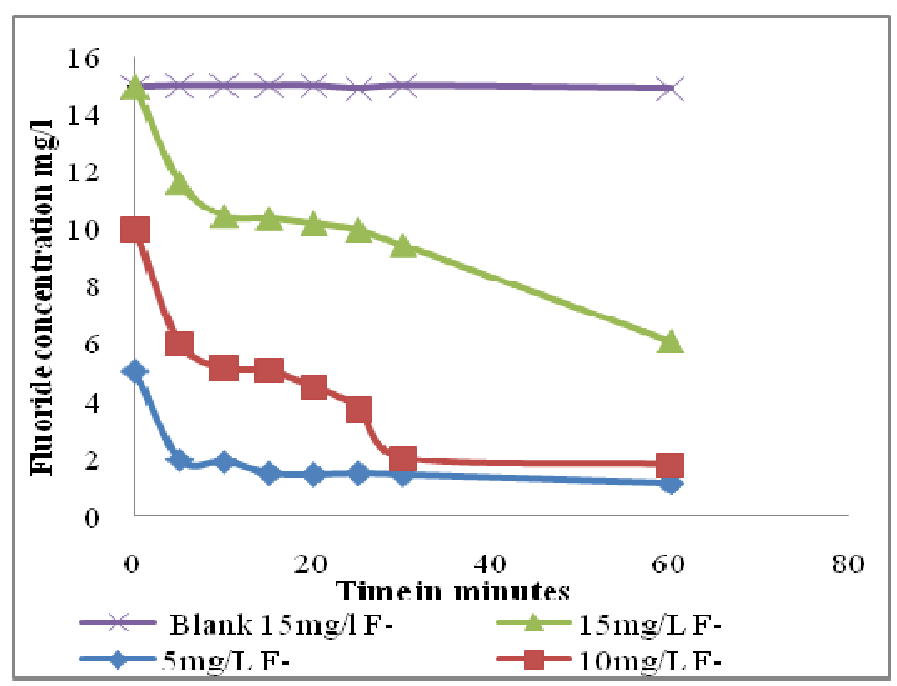

Fig. 5: Fluoride removal with HDTMA-Br modified zeolite under controlled pH conditions

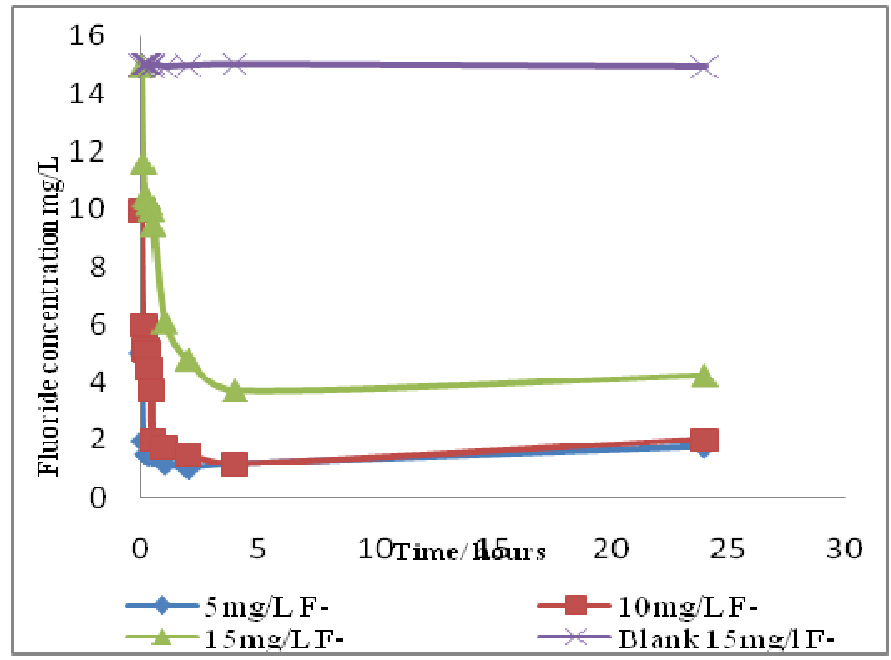

Fig. 6: Fluoride removal using HDTMA-Br modified zeolite under controlled pH conditions over a period of 24 hours 
continued until between the 30th and 60th minute where a near equilibrium was reached.

The rapid uptake of fluoride at the beginning of the adsorption process can be attributed to the rapid attachment of fluoride ions to the surface of the surfactant modified zeolite. The gradual reduction in adsorption could be due to the decrease in adsorption sites.

It was observed that as the initial fluoride concentration was increased from $5 \mathrm{mg} / \mathrm{L}$ to $15 \mathrm{mg} / \mathrm{L}$, the amount of fluoride adsorbed decreased (Fig. 6). At a lower fluoride concentration, most of the fluoride ions present in the model water would interact with the adsorption sites on the adsorbent and be adsorbed. However, at a higher concentration, the adsorption sites become quickly saturated because more fluoride ions are available. Besides for a fixed adsorbent dose the available sites for adsorption are limited. Similar trends have been reported for fluoride removal by Bhaumik et al. (2012) using eggshell powder.

\section{CONCLUSIONS}

Kaolin was successfully transformed into highly crystalline zeolite Na-LSX by alkaline fusion followed by hydrothermal treatment and modification with the surfactant hexadecyltrimethylammonium bromide. Characterization results show the synthesized zeolite as zeolite Na-LSX withOxygen(60\%), Silicon (15\%) and Aluminum $(13 \%)$ as the predominant elements in their crystal structure that exhibits octahedral morphology. The adsorption studies showed that the unmodified zeolite could not adsorb fluoride ions. The surfactant HDTMA-Br modified zeolite was able to remove the fluoride significantly from aqueous medium. Fluoride adsorption was found to be $\mathrm{pH}$ dependent and the maximum removal of fluoride was obtained at $\mathrm{pH}$ 6.0- 7.0.

\section{REFERENCES}

Bhaumik, R., Mondal, N. K., Das, B., Roy, P., Pal, K. C., Das, C., Banerjee, A. and Datta, J. K. (2012). Eggshell Powder as an Adsorbent for Removal of Fluoride from Aqueous Solution: Equilibrium, Kinetic and Thermody- namic Studies. E-Journal of Chemistry, 9 (3):1457-1480.

Cobbina, S. J., Nyame, F. K. and Obiri, S., (2012). Groundwater Quality in the Sahelian Region of Northern Ghana, West Africa. Research Journal of Environmental and Earth Sciences, 4(4): 482-491.

Kaur, J., Bhunia, H. and Rajor, A. (2013). Removal of fluoride from drinking water by activated carbon. VSRD International Journal of Technical and Non-Technical Research, 5(8): 187-192.

Keka, O., Narayan, C. P. and Amar, N. S. (2004). Zeolite from fly ash: synthesis and characterization, Bulletin of Material Science, 27(6): 555-564.

Mohammadi A., Bina B., Ebrahimi A., Hajizadeh Y., Amin M. M. and Pourzamani H. (2012). Effectiveness of nanozeolite modified by cationic surfactant in the removal of disinfection by-product precursors from water solution. International Journal of Environmental Health Engineering, 1(1): 14-19.

Murugan, M. and Subramanian, E. (2006). Studies on defluoridation of water by Tamarind seed, an unconventional biosorbent. Journal of Water and Health, 4(4): 453-461.

Renuka, P. and Pushpanjali, K. (2013). Review on Defluoridation Techniques of Water, The International Journal of Engineering and Science (Ijes), 2 (3): 86-94.

Reyes, C. A. R. and Fiallo, L. Y. V. (2011). Application of Illite- and Kaolinite-Rich Claysin the Synthesis of Zeolites for Wastewater Treatment, In: Dar, I. A., ed., Earth and Environmental Sciences, InTech, pp. 363-374. Available from: http://www.intechopen.com/ books/earth-and-environmental-sciences/ application-of-illite-and-kaolinite-rich-claysin-the-synthesis-of-zeolites-for-wastewatertreatment. 
Defluorination of drinking water ... 21

Shoumkova, A. (2011). Zeolites for water and wastewater treatment: An overview. Accessed on 26-07-2013 from http://www.ausihem.org/ web_documents/2011_si_shoumkova.pdf 